\title{
Psychopathology and cognition in children with 22q11.2 deletion syndrome
}

Maria Niarchou, Stanley Zammit, Stephanie H. M. van Goozen, Anita Thapar, Hayley M. Tierling, Michael J. Owen and Marianne B. M. van den Bree

\section{Background}

Children with 22q11.2 deletion syndrome (22q11.2DS) have been reported to have high rates of cognitive and psychiatric problems.

\section{Aims \\ To establish the nature and prevalence of psychiatric disorder and neurocognitive impairment in children with 22q11.2DS and test whether risk of psychopathology is mediated by the children's intellectual impairment.}

\section{Method}

Neurocognition and psychopathology were assessed in 80 children with 22q11.2DS (mean age 10.2 years, s.d. $=2.1$ ) and 39 sibling controls (mean age 10.9 years, s.d. $=2.0)$.

\section{Results}

More than half (54\%) of children with 22q11.2DS met diagnostic criteria for one or more DSM-IV-TR psychiatric disorder. These children had lower IQ (mean 76.8, s.d. $=13.0$ ) than controls (mean 108.6, s.d. $=15.2)(P<0.001)$ and showed a range of neurocognitive impairments. Increased risk of psychopathology was not mediated by intellectual impairment.

\section{Conclusions}

22q11.2DS is not related to a specific psychiatric phenotype in children. Moreover, the deletion has largely independent effects on IQ and risk of psychopathology, indicating that psychopathology in 22q11.2DS is not a non-specific consequence of generalised cognitive impairment.

\section{Declaration of interest}

None. 22q11.2 Deletion syndrome (22q11.2DS) is one of the most common genetic causes of intellectual disability and psychopathology ${ }^{1}$ and one of the strongest known risk factors for developing schizophrenia in adulthood. ${ }^{2,3}$ Studies have shown that children with 22q11.2DS have high rates of psychopathology, especially attention-deficit hyperactivity disorder (ADHD), anxiety disorders and oppositional defiant disorder (ODD), and a range of neurocognitive problems (e.g. Green et al, ${ }^{4}$ Shashi et al, ${ }^{5}$ Hooper et $\left.a l^{6}\right)$. There are, however, considerable discrepancies in the reported rates of psychiatric disorders stemming from the difficulties associated with recruiting large sample sizes at similar developmental stages in such a rare syndrome. Moreover, a specific neurocognitive profile has not been identified. ${ }^{7}$ It is also not clear whether intellectual disability (as assessed by an IQ test) in children with 22q11.2DS influences performance on other cognitive measures and whether the pattern of associations between cognitive tests is similar to this of a sample with uncompromised cognitive functions. It also remains unclear to what extent psychopathology in children with 22q11.2DS is a direct consequence of their deletion (Fig. 1, Model A) or whether this relationship is better explained by the children's intellectual impairment (Fig. 1, Model B). ${ }^{8,9}$ That is, does the deletion increase risk of intellectual impairment, which subsequently predisposes to risk of psychopathology? If this were the case, it might be argued that psychopathology in 22q11.2DS is a non-specific consequence of generalised intellectual impairment. Or, alternatively, does the deletion predispose independently to both cognitive impairments and psychopathology? This would imply that cognitive impairments index genetic risk factors for psychiatric disorders, rather than mediate the risk of psychopathology.

The primary goals of this study were twofold. First, to explore differences in the prevalence and nature of psychiatric and cognitive problems in children with 22q11.2DS compared with their siblings. As a secondary analyses, we also explored the relations between IQ and other cognitive measures as well as whether the pattern of associations between the cognitive measures is similar for children with 22q11.2DS and their siblings. Second, to examine whether any association between deletion status and psychopathology is mediated through intellectual impairment. To address these issues we conducted one of the largest studies of children with 22q11.2DS and sibling controls to date and one of the few studies to have included both detailed psychiatric and cognitive assessments. Based on preliminary findings on other copy number variants $(\mathrm{CNVs}),{ }^{9}$ we hypothesised that IQ would not mediate the relationship between the deletion and psychiatric problems (Model A., Fig. 1). ${ }^{9}$ As an exploratory analysis, we also examined whether any other neurocognitive deficits mediated this relationship.

\section{Method}

\section{Participants}

As part of the Experiences of CHildren with cOpy number variants (ECHO) study, 80 children $(50 \%$ females $)$ with 22q11.2DS aged 6.6-14.1 years (mean 10.2, s.d. $=2.1$ ) and 39 sibling controls (56\% females) aged 6.3-14.3 years (mean 10.9, s.d. $=2.0$ ) were recruited from 14 genetics clinics across the UK, the British 22q11 deletion syndrome charities Max Appeal! and The 22Crew, and our ECHO study website (http://medicine. cardiff.ac.uk/psychological-medicine-neuroscience/areas-research/ copy-number-variant-research/research-projects/).

Table 1 provides a sociodemographic description of the sample. Numbers of children differ for different measures as five children with 22q11.2DS were not able to complete all the cognitive assessments. Furthermore, due to geographical considerations we did not conduct any neurocognitive testing in six children (in these cases psychiatric interviews were conducted using video-call).

Presence of the deletion was confirmed for all children with 22q11.2DS $(87.5 \%$ of families were recruited from medical 
Model A

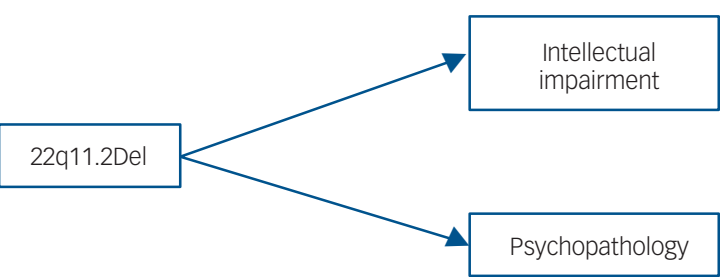

Model B

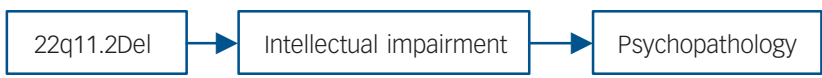

Mediation model

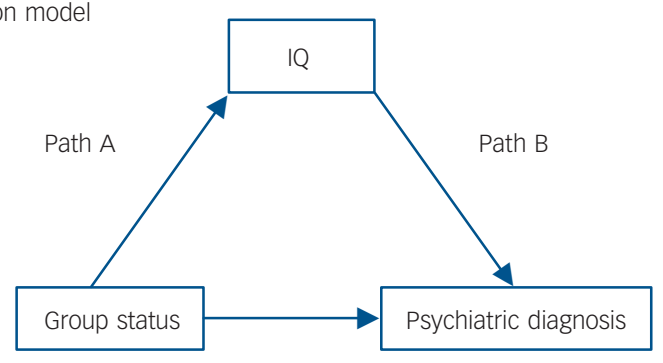

Path C

Fig. 1 Representation of possible relationships between intellectual impairment and psychopathology and the $22 q 11.2$ deletion.

According to Model A, psychopathology is a direct consequence of the deletion, whereas according to Model B, psychopathology can be better explained by th intellectual impairment caused by the 22q11.2 deletion. The mediation model examines the role of group status (children with 22q11.2DS V. sibling controls) on psychiatric diagnosis through IQ. Del, deletion.

genetics clinics and for the remaining $12.5 \%$ medical records were made available by the families).

Informed written consent was obtained prior to recruitment from the carers of the children and recruitment was carried out in agreement with protocols approved by the appropriate research National Health Service ethics and research and development committees.

\section{Psychopathology}

Psychopathology was assessed with the Child and Adolescent Psychiatric Assessment (CAPA) ${ }^{10}$ by means of semi-structured interview with the primary caregiver. In the section of the interview dealing with psychotic experiences, initial screening questions probed for any evidence of perceptual disorders or hallucinations, delusions or psychotic abnormalities of thought processes. If these screening questions were answered affirmatively, the interviewer continued with more detailed questions about the nature of possible symptoms. Specifically, questions are included regarding content and location of auditory, olfactory and tactile hallucinations. Questions were asked about thought insertion and broadcast, thought echo and withdrawal and delusional thinking. Phenomena not coded as psychotic symptoms included hypnagogic/pompic hallucinations, eidetic imagery, elaborated fantasies, imaginary companions, illusions, hallucinations occurring as part of a seizure or clouded sensorium, spots/stripes before the eyes and sensory changes associated with headaches. Psychotic experiences were also assessed by children's self-reports using the same instrument. All interviews were conducted by trained psychologists, who were supervised by a consultant child and adolescent psychiatrist. Interviews were

\begin{tabular}{|c|c|}
\hline & $\%$ \\
\hline \multicolumn{2}{|l|}{ Family ethnic background } \\
\hline European & 91.25 \\
\hline Mixed & 6.25 \\
\hline Non-European & 2.5 \\
\hline \multicolumn{2}{|l|}{ Highest maternal educational qualification } \\
\hline Low: O-levels or GCSES & 38.75 \\
\hline Middle: A-levels/highers or vocational training & 40 \\
\hline High: university degree and/or other higher & \\
\hline postgraduate qualification & 20 \\
\hline Unknown & 1.25 \\
\hline \multicolumn{2}{|l|}{ Family income, $f$} \\
\hline$\leqslant 19999$ & 16.25 \\
\hline $20000-39000$ & 27.5 \\
\hline $40000-59000$ & 18.75 \\
\hline$\geqslant 60000$ & 25 \\
\hline Unknown & 12.5 \\
\hline
\end{tabular}

audiotaped for monitoring and assignment of DSM-IV-TR ${ }^{11}$ diagnoses by the child and adolescent psychiatrist. We did not regard ADHD, ODD, pervasive developmental disorder and mood and anxiety disorders as mutually exclusive diagnoses.

Autistic behaviour was assessed using the Social Communication Questionnaire (SCQ), ${ }^{12}$ which was completed by the primary caregiver. Total scores can range from 0 to 39. The cut-off of 15, which is suggestive of autism spectrum disorder (ASD), was used in our analyses.

With regard to psychotropic medication, one child with 22q11.2DS and one sibling were receiving oral methylphenidate for ADHD.

\section{Cognition}

Neurocognitive assessments were conducted by trained raters. An estimate of general intelligence (IQ) was obtained by administering the Wechsler Abbreviated Scale of Intelligence (WASI; four subtests). ${ }^{13}$ Processing speed was assessed with the Reaction Time (RTI) (child mode) test; attention and vigilance were assessed with the Match to Sample visual search (MTS) and the Rapid Visual Information Processing (RVP) (child mode) tests, from the CANTAB (Cambridge Neuropsychological Automated Battery eclipse version 3 software, Cambridge, 2006); executive function was assessed with the Spatial Working Memory (SWM) and the Stockings of Cambridge (SOC) tests from the CANTAB. To obtain a global assessment of executive function we also administered the Wisconsin Card Sorting Test (WSCT). ${ }^{14}$

\section{Data analysis}

Data analysis was conducted using Stata (version 11) for Windows XP.

\section{Psychopathology (Goal 1)}

The prevalence of psychiatric problems in children with 22q11.2DS compared with their siblings was examined using a $\chi^{2}$-test. Associations between age and psychiatric diagnoses were examined using Spearman's rank correlations, and association between gender and psychiatric diagnoses were examined using $\chi^{2}$-tests. To examine whether any of the comorbid diagnoses occurred more frequently than others, $\chi^{2}$-tests were used.

\section{Nature and prevalence of cognitive impairments (Goal 1)}

All neurocognitive measures were standardised to have a mean of zero and a standard deviation of one, apart from the 'failure to 
maintain set' variable of the WCST (which is categorical) and IQ score. Apart from IQ, all other cognitive variables were not normally distributed. To compare the mean differences in cognitive performance of the children with 22q11.2DS with their siblings, $t$-tests or, in the case of non-normally distributed cognitive variables, Mann-Whitney $U$-tests were conducted. Spearman's rank correlations were used to estimate the correlations between IQ and age, and IQ and gender. A $t$-test was used to examine whether the average verbal IQ in children with 22q11.2DS was different from the average performance IQ.

\section{IQ in relation to other cognitive measures}

\section{and patterns of associations (Goal 1)}

Spearman's rank correlations were used to estimate the correlations between IQ and other neurocognitive measures. A comparison of the magnitudes of the correlations of the neurocognitive tests for children with 22q11.2DS $v$. sibling controls was conducted following Fisher's r-to-z transformation. ${ }^{15}$ A two-group variance-comparison test using the sdtest command was employed to examine whether there was greater variation in neurocognitive performance in the children with 22q11.2DS in relation to their siblings.

\section{Psychopathology and cognition (Goal 2)}

In order to determine whether the differences in psychiatric diagnoses between the children with 22q11.2DS and their sibling controls could be explained by IQ, we performed the following analyses. Independent regressions were performed in the total sample to determine whether the independent variable (i.e. IQ score) predicted presence of psychiatric diagnosis $(0=$ no diagnosis, $1=$ present diagnosis). From these regressions we derived odds ratios (ORs) and 95\% confidence intervals (CIs). To facilitate interpretation of the results, IQ score was standardised to have a mean of 0 and a standard deviation of 1 and recoded so that higher score indicated worse performance. These analyses were conducted only for the psychiatric diagnoses that were significantly different between the two groups: 1. ADHD (absent (0) $v$. present (1)); 2. any anxiety disorder (absent (0) $v$. present (1)); 3. ODD (absent (0) v. present (1)); 4. ASD screening $(0=$ SCQ score $<15,1=$ SCQ score $\geqslant 15)$. To test the assumption that the relationship between IQ and psychopathology was similar in children with 22q11.2DS and siblings and that combining the two groups for subsequent analysis was therefore appropriate, we assessed homogeneity of ORs using the $\chi^{2}$-test of homogeneity. Odds ratios and 95\% confidence intervals for the associations between IQ and psychopathology after adjusting for group status (22q11.2DS $v$. siblings) were estimated using the Mantel-Haenszel method. ${ }^{16}$

Mediation analysis was also conducted to examine the extent to which IQ score mediated the associations between group status and psychiatric diagnosis. Figure 1 (Model B) illustrates a representation of the model tested. The binary_mediation command was used to estimate the indirect effect of IQ on psychopathology, using the product of coefficients approach, which is considered to have good power and low type I error. ${ }^{17}$ Standard errors and biased-corrected confidence intervals were also calculated using bootstrapping with 5000 replications. We repeated these analyses to explore whether the other cognitive measures mediated the relation between the deletion and psychiatric problems. To limit the number of statistical comparisons we examined only the primary scores of these cognitive tests (i.e. MTS total correct; RTI 5-choice reaction time; RVP A'; SOC problems solved in minimum moves; and SWM between errors score, number of perseverative errors).

\section{Results}

\section{Psychopathology (Goal 1)}

More than half (54\%) of the children with 22q11.2DS met diagnostic criteria for one or more DSM-IV-TR psychiatric disorders, compared with $10 \%$ of the siblings, $P<0.001$ (Table 2). Children with 22q11.2DS exhibited higher rates of ADHD, ODD and anxiety disorders in relation to their siblings. Furthermore, more children with 22q11.2DS (26\%) met the cut-off for probable ASD diagnosis in relation to their siblings (5\%). Psychiatric diagnoses were not related to age or gender in the children with 22q11.2DS (data not presented). High rates of comorbidity were observed for children with 22q11.2DS (Fig. 2): 12 (37.5\%) children with ADHD also had at least one anxiety disorder; 12

\begin{tabular}{|c|c|c|c|c|c|}
\hline \multirow[b]{2}{*}{ Diagnosis } & \multicolumn{2}{|c|}{ Children with 22q11.2DS } & \multicolumn{2}{|c|}{ Siblings } & \multirow[b]{2}{*}{$P$} \\
\hline & $n(\%)$ & $95 \% \mathrm{Cl}, \%$ & $n(\%)$ & $95 \% \mathrm{Cl}, \%$ & \\
\hline Any diagnosis & $43(54.5)$ & $44-64$ & $4(10.5)$ & $6-19$ & $<0.001$ \\
\hline Any anxiety disorder & $21(26.3)$ & $18-36$ & $2(5.3)$ & $2-11$ & 0.01 \\
\hline Generalised anxiety disorder & $5(6.3)$ & $2-13$ & 0 & $0-4$ & 0.12 \\
\hline Panic disorder without agoraphobia & $1(1.3)$ & $0-6$ & 0 & $0-4$ & 0.48 \\
\hline Panic disorder with agoraphobia & $2(2.5)$ & $0-9$ & 0 & $0-4$ & 0.33 \\
\hline Agoraphobia without history of panic disorder & $1(1.3)^{a}$ & $0-6$ & 0 & $0-4$ & 0.49 \\
\hline Specific phobia & $12(15.2)^{\mathrm{a}}$ & $9-24$ & $1(2.6)$ & $0-9$ & 0.04 \\
\hline Social phobia & $14(17.5)$ & $11-27$ & $2(5.3)$ & $2-11$ & 0.07 \\
\hline Obsessive-compulsive disorder & $3(3.8)$ & $1-10$ & 0 & $0-4$ & 0.23 \\
\hline Separation anxiety disorder & $2(9.1)$ & $4-16$ & 0 & $0-4$ & 0.41 \\
\hline Attention-deficit hyperactivity disorder & $32(40.5)^{a}$ & $40-60$ & $2(5.3)$ & $2-11$ & $<0.001$ \\
\hline Oppositional defiant disorder & $15(18.8)$ & $12-28$ & $0^{\mathrm{a}}$ & $0-4$ & 0.005 \\
\hline Tic disorder & $6(7.5)$ & $4-15$ & $1(2.7)^{\mathrm{a}}$ & $0-9$ & 0.31 \\
\hline Delusional disorder & $2(2.5)$ & $0-9$ & 0 & $0-4$ & 0.33 \\
\hline Selective mutism & $1(1.3)$ & $0-6$ & 0 & $0-4$ & 0.49 \\
\hline Autism spectrum disorder screening ${ }^{b}$ & $20(26.0)$ & $18-36$ & $2(5.3)$ & $2-11$ & 0.01 \\
\hline
\end{tabular}




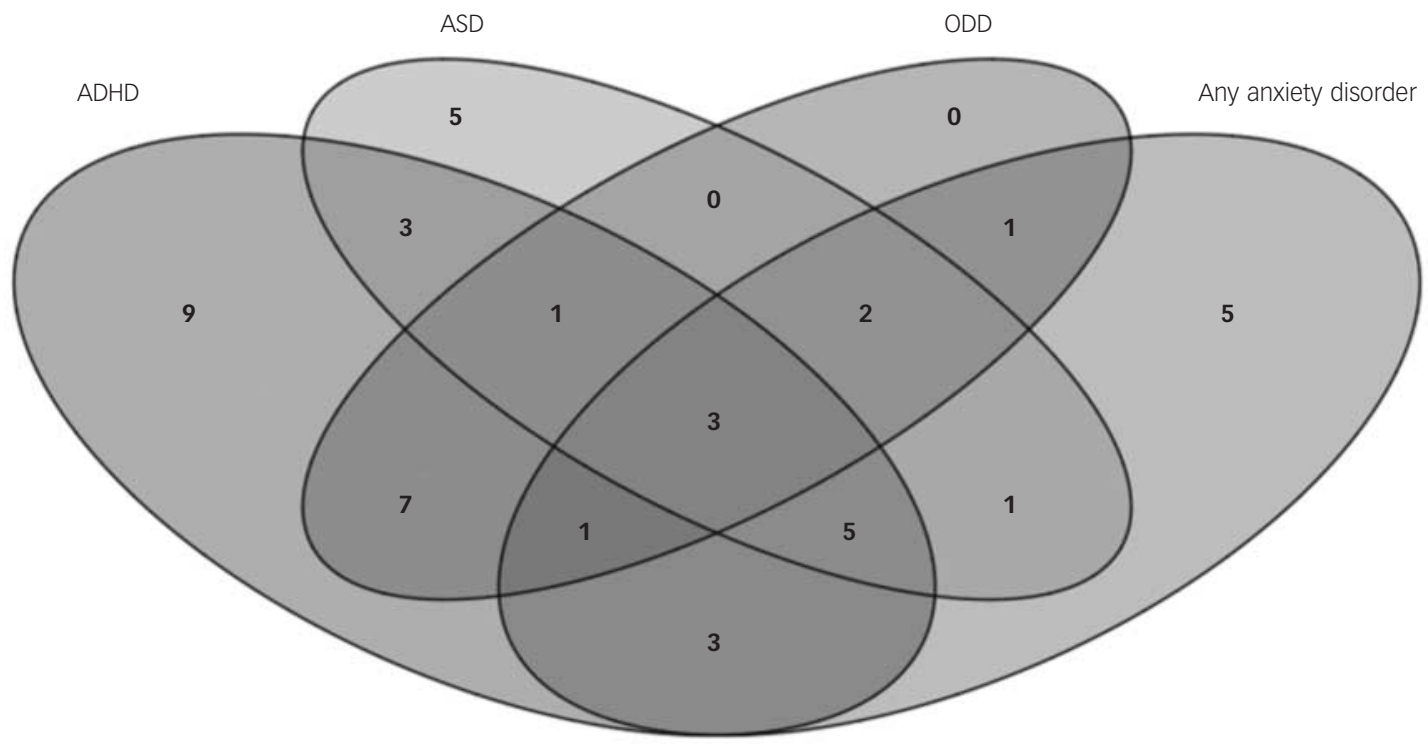

Fig. 2 Comorbidity in children with 22q11.2 deletion syndrome.

ADHD, attention-deficit hyperactivity disorder; ASD, autism spectrum disorder; ODD, oppositional defiant disorder.

(37.5\%) had ODD; and $12(41.2 \%)$ screened positive for probable ASD. Among children with an anxiety disorder, 7 (33.3\%) also had ODD and 11 (52.4\%) screened positive for probable ASD. Among children with ODD, $6(30 \%)$ also screened positive for probable ASD. None of the comorbid diagnoses occurred more frequently than others $(P>0.2)$. Overall, psychotic experiences (parent and self-report combined) were reported by $10 \%$ of children with 22q11.2DS, and $8 \%$ of their siblings. Parents reported psychotic experiences for 5\% of the children with 22q11.2DS and none of their siblings, whereas $6 \%$ of the children with 22q11.2DS and $8 \%$ of their siblings self-reported psychotic experiences. Parental and child reports were in agreement for only one child (who had 22q11.2DS).

\section{Nature and prevalence of cognitive impairments (Goal 1)}

Children with 22q11.2DS had a lower estimated total IQ and performed worse than their siblings on all cognitive tests (Table 3 ). The IQ distribution of the children with 22q11.2DS was shifted over 30 points to the left compared with the siblings (Fig. 3). Of the children with $22 \mathrm{q} 11.2,30.6 \%$ had mild intellectual disability (IQ range 53-69), 30.6\% had a borderline IQ score (70-79) and $38.9 \%$ had an average IQ score (81-109). Older children with 22q11.2DS had lower IQs than younger children $(r=-0.32$, $P=0.01)$ but this was not the case for siblings $(r=-0.09$, $P=0.61$ ). No gender differences were found for IQ between children with 22q11.2DS and their siblings. There were no differences between verbal and performance IQ for children with 22q11.2DS $(t=-0.24, P=0.82)$.

We found $22 \%$ of children with 22q11.2DS had higher verbal $(\geqslant 10$ points) than performance IQ, but equally $22 \%$ had higher performance $(\geqslant 10$ points) than verbal IQ. Children with 22q11.2DS were also found to perform worse than their siblings on all other neurocognitive tests (Table 3).

\section{IQ in relation to the other cognitive measures and patterns of associations (Goal 1)}

Correlation analysis (Table 4) indicated that the CANTAB measures and the WCST did tap into neurocognitive domains that were independent of IQ in children with 22q11.2DS. As a sensitivity analysis, we reran the analyses comparing cognitive performance in children with 22q11.2DS and sibling controls, including only children with 22q11.2DS with IQ $>70$ and results remained substantially the same, suggesting the findings were not driven by the subgroup with mild intellectual disability. The pattern of associations between the neurocognitive tests ranged from low to modest in strength (Table 5), indicating that these cognitive tests do measure different cognitive abilities in both groups of children. Although the correlations between the tests tended to be somewhat higher for children with 22q11.2DS (range 0.15 to 0.55$)$ compared with sibling controls $(-0.17$ to 0.44$)$, formal comparison indicated the magnitude of associations was higher in children with 22q11.2DS for only 2 out of 15 correlations: RVP test with both the RTI test and perseverative

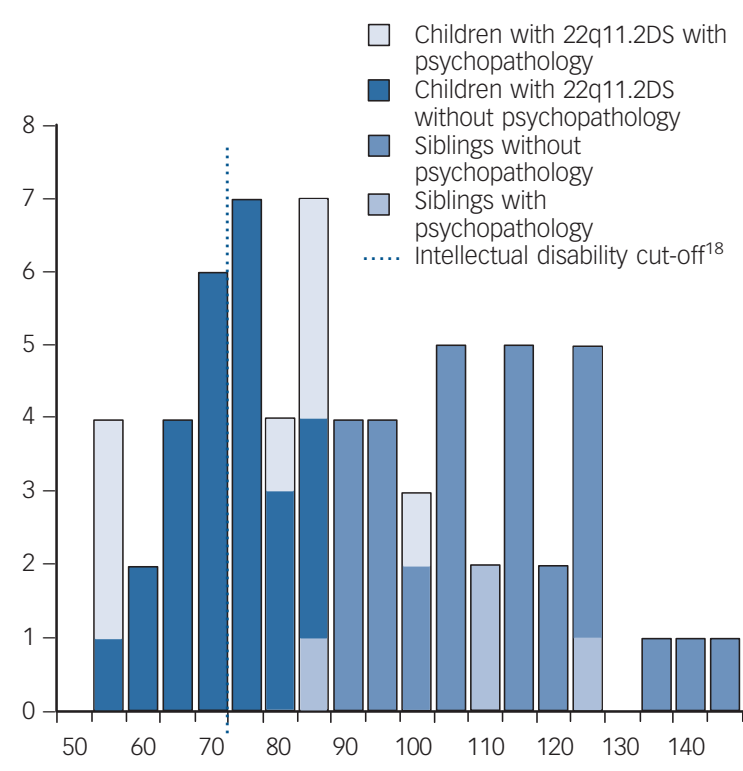

Fig. 3 IQ distributions and psychopathology of children with 22q11.2 deletion syndrome and their siblings. 
errors of the WCST. The RTI test showed the highest correlations not only with the measures within the attention domain (e.g. RVP test) but also with all the measures of executive function (e.g. SOC test) for children with 22q11.2DS. Finally, we found larger standard deviations for most cognitive tests indicating greater variation in the cognitive performance of children with 22q11.2DS in relation to their siblings, with the exception of IQ and SWM tests (Table 6).

\section{Psychopathology and cognition (Goal 2)}

Initial analyses in the total sample of children with 22q11.2DS and their siblings showed a relationship between IQ and risk for psychopathology (i.e. ADHD diagnosis, any anxiety disorder, and ASD screening) (Table 7). This analysis could not be conducted for ODD because none of the siblings met criteria for this diagnosis. In analyses stratified by group status there was no evidence of association between IQ and psychopathology either in children with 22q11.2DS or their siblings. In the mediation analyses, with IQ as the mediator, the indirect effects via IQ (Fig. 1, Mediation model, paths A and B) were considerably weaker than the direct effects between group status and psychopathology (path C) (Table 8). Similar results were obtained when regression and mediation analyses were conducted for the other cognitive measures (data not presented). The only exception was an indirect effect for the SOC task component problems solved in minimum moves $(-12,95 \% \mathrm{CI}-0.25$ to -0.009$)$ and ASD. However, given the number of tests in our exploratory analysis $(n=21)$, this effect did not survive correction for multiple testing.

\section{Discussion \\ Psychopathology}

More than half (54\%) of children with 22q11.2DS met diagnostic criteria for one or more DSM-IV-TR psychiatric disorder. They had higher rates of ADHD, ASD, ODD and anxiety disorders compared with sibling controls. These rates are broadly similar to those reported in previous studies of children with 22q11.2DS (e.g. Green et $a l^{4}$ ) and to population studies of children with intellectual disabilities (e.g. Emerson ${ }^{19}$ ), indicating that in childhood, 22q11.2DS does not seem to be related to a specific psychiatric phenotype, ${ }^{7}$ at least as captured by current diagnostic systems. ${ }^{20}$ It also suggests that these disorders can share underlying pathogenic mechanisms with intellectual disability. Comorbidity was also substantial, providing further evidence that the effect of the deletion is widespread across a range of neurodevelopmental syndromes. This is in keeping with previous studies of children with 22q11.2DS (e.g. James et $a l^{21}$ ), studies of rare CNVs and single-gene disorders which similarly tend to be associated with increased risk of many different types of psychopathology (e.g. Miller et $a l^{22}$ ).

The frequency of psychotic-like experiences was similar between children with 22q11.2DS and their siblings, indicating that at this age children with 22q11.2DS are too young to exhibit the higher rates of psychotic experiences robustly seen in late adolescence and adulthood. Interestingly, parent and child reports were in agreement only for one child with 22q11.2DS and none of the siblings for whom any psychotic experiences were only by self-report. This could be because children are not willing to share these symptoms with their parents or due to difficulties associated with obtaining reliable data on psychotic experiences in children of this age.

\section{Cognition}

Our findings demonstrate that the IQ distribution of the children with 22q11.2DS is shifted over 30 points to the left in relation to

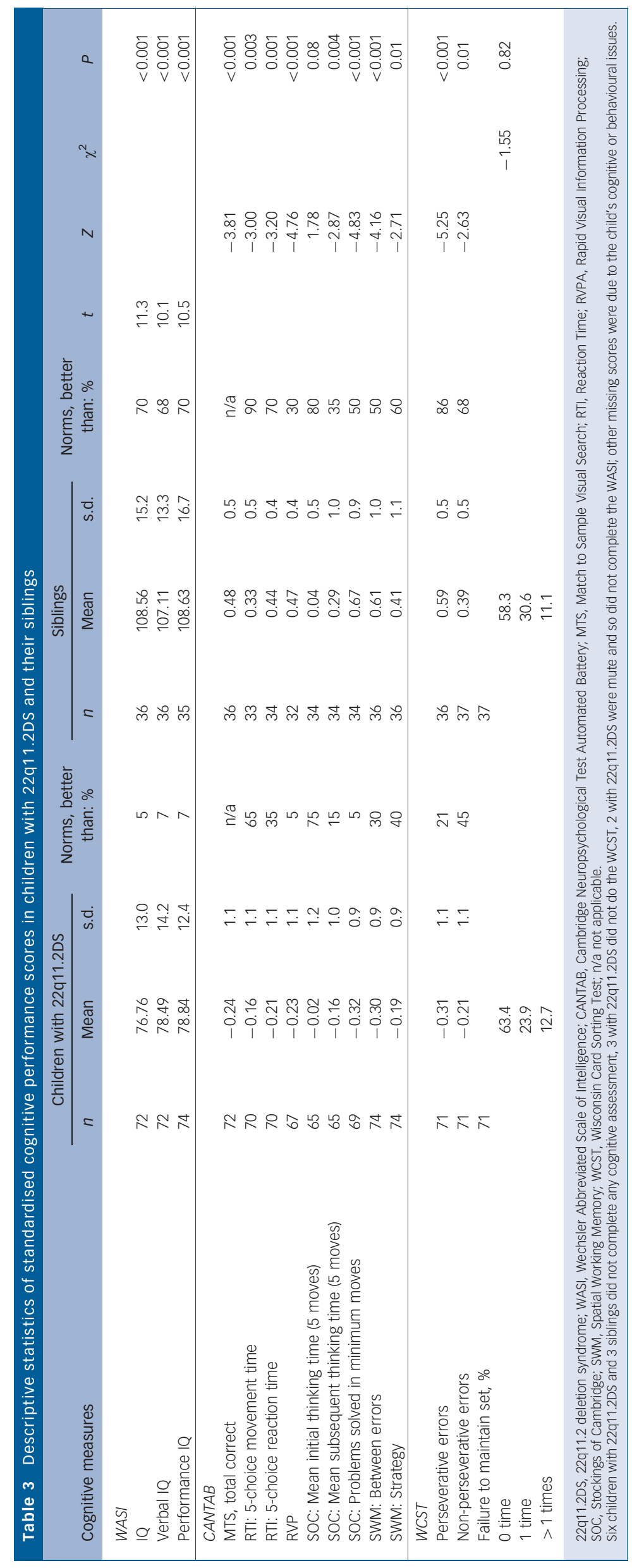




\begin{tabular}{|c|c|c|}
\hline \multirow[b]{2}{*}{ Cognitive measure } & \multicolumn{2}{|c|}{ IQ } \\
\hline & rho & $P$ \\
\hline MTS, total correct & 0.09 & 0.45 \\
\hline \multicolumn{3}{|l|}{ RTI } \\
\hline 5-choice movement time & 0.12 & 0.32 \\
\hline 5-choice reaction time & 0.07 & 0.59 \\
\hline RVPA & 0.20 & 0.10 \\
\hline \multicolumn{3}{|l|}{ SOC } \\
\hline Mean initial thinking time & -0.04 & 0.75 \\
\hline Mean subsequent thinking time & 0.08 & 0.54 \\
\hline Problems solved in minimum moves & 0.16 & 0.20 \\
\hline \multicolumn{3}{|l|}{ SWM } \\
\hline Between errors & 0.35 & 0.003 \\
\hline Strategy & 0.35 & 0.003 \\
\hline \multicolumn{3}{|l|}{ WCST } \\
\hline Perseverative errors & 0.25 & 0.04 \\
\hline Non-perseverative errors & -0.13 & 0.29 \\
\hline Failure to maintain set & -0.07 & 0.57 \\
\hline
\end{tabular}

their siblings. This is in agreement with previous studies in $22 \mathrm{q} 11.2 \mathrm{DS}^{6}$ as well as the literature on single-gene disorders (e.g. fragile-X syndrome $e^{23}$ ) and other neurodevelopmental syndromes (e.g. ASD and $\mathrm{ADHD}^{24}$ ). Even though performance on the neurocognitive tests was generally impaired in the entire sample of children with 22q11.2DS, it was also variable. That is, performance on IQ was unrelated to performance on most cognitive tests, a finding in accordance with reports in other neurodevelopmental disorders (e.g. $\mathrm{ADHD}^{25}$ ). Furthermore, among the neurocognitive tests, performance in one domain did not represent a good indicator of performance in another domain (as evidenced by modest correlation coefficients). However, for children with 22q11.2DS performance on the RVP task (an indicator of attention) was more strongly associated with performance on the RTI test (an indicator of processing speed) as well as with the number of perseverative errors from the WCST (an indicator of executive function) than for their siblings. Generally, the five-choice RTI score (an indicator of processing speed) showed the highest correlations (0.25-0.55) for children with $22 \mathrm{q} 11.2 \mathrm{DS}$, not only with measures within the domain of attention, but also with all measures of executive function. This is in accordance with the existing literature that underlines the relevance and importance of processing speed to both typical and atypical development. For example, several studies in typically developing children show that: processing speed mediates the developmental course of other cognitive abilities, such as working memory and response inhibition; ${ }^{26}$ cognitive slowing might restrict performance in other cognitive abilities such as executive functions; ${ }^{26}$ among neurocognitive tests, processing speed best predicts psychotic experiences. ${ }^{27}$ Furthermore, children with 22q11.2DS had greater variation in cognitive performance in relation to their siblings, a finding in agreement with previous reports in 22q11.2DS. ${ }^{28}$ This variation could be due to the deletion having widespread effects in cognitive performance but we cannot exclude the possibility of other confounding factors, such as more attention or behavioural problems in children with 22q11.2DS in relation to their siblings, which might have influenced their performance in the cognitive tests. We did not find evidence of better verbal than non-verbal abilities in line with some ${ }^{29}$ but not all previous studies. ${ }^{30}$ Moreover, although previous studies have shown discrepancies between verbal and performance IQ (usually between 8 to 10 points $^{31}$ ), the number of children with better verbal than performance IQ ( $\geqslant 10$ points) in our study was similar to the number with better performance than verbal IQ ( $\geqslant 10$ points). Therefore our study does not support the hypothesis that 22q11.2DS is specifically associated with non-verbal intellectual disability.

\section{Lack of associations between cognitive impairment and psychopathology}

There has been disagreement on whether there is a relationship between IQ and psychopathology in 22q11.2DS with some studies reporting relationships, ${ }^{30}$ while others do not. ${ }^{32}$ In line with our predictions, there was no relation between psychopathology and IQ in children with 22q11.2DS.

The availability of a sample of sibling controls in which the same assessments were conducted as in the children with 22q11.2DS, in combination with a relatively large sample size, allowed us to conduct the first mediation analysis for this syndrome. The findings showed that psychopathology in 22q11.2DS is not explained by indirect effects, mediated by the children's intellectual impairment.

We also explored the associations with other neurocognitive measures and our results indicated only an indirect effect of the deletion on probable ASD diagnosis through the SOC (an indicator of planning) test. This effect, however, was in the opposite direction than expected and therefore, given the exploratory nature of our analysis, we interpreted this result as

\begin{tabular}{|c|c|c|c|c|c|c|c|c|c|c|}
\hline \multirow[b]{2}{*}{$\begin{array}{l}\text { Cognitive } \\
\text { measure }\end{array}$} & \multicolumn{2}{|l|}{ MTS } & \multicolumn{2}{|l|}{ RTI } & \multicolumn{2}{|c|}{ RVPA } & \multicolumn{2}{|l|}{ SOC } & \multicolumn{2}{|c|}{ SWM } \\
\hline & $\begin{array}{l}\text { Children with } \\
22 \mathrm{q} 11.2 \mathrm{DS}\end{array}$ & Siblings & $\begin{array}{l}\text { Children with } \\
\text { 22q11.2DS }\end{array}$ & Siblings & $\begin{array}{l}\text { Children with } \\
\text { 22q11.2DS }\end{array}$ & Siblings & $\begin{array}{l}\text { Children with } \\
\text { 22q11.2DS }\end{array}$ & Siblings & $\begin{array}{c}\text { Children with } \\
\text { 22q11.2DS }\end{array}$ & Siblings \\
\hline MTS, total correct & 1 & 1 & & & & & & & & \\
\hline RTI, 5-choice reaction time & 0.28 & 0.07 & 1 & 1 & & & & & & \\
\hline RVPA & 0.33 & -0.07 & 0.55 & 0.14 & 1 & 1 & & & & \\
\hline $\begin{array}{l}\text { SOC, problems solved in } \\
\text { minimum moves }\end{array}$ & 0.18 & -0.12 & 0.35 & 0.35 & 0.28 & 0.19 & 1 & 1 & & \\
\hline SWM, between errors & 0.15 & -0.17 & 0.26 & 0.20 & 0.25 & 0.44 & 0.28 & 0.26 & 1 & 1 \\
\hline WCST, perseverative errors & 0.16 & 0.02 & 0.25 & 0.09 & 0.27 & -0.18 & 0.21 & 0.41 & 0.16 & -0.01 \\
\hline
\end{tabular}


Table 6 Results from two-group variance-comparison test in standardised cognitive performance between children with $22 \mathrm{q} 11.2$ deletion syndrome (22q11.2DS) and their sibling controls

\begin{tabular}{|c|c|c|c|c|}
\hline Cognitive measures & Children with 22q11.2DS, s.d. & Siblings, s.d. & $F$ & $P$ \\
\hline \multicolumn{5}{|l|}{ WASI } \\
\hline IQ & 13.0 & 15.0 & 1.36 & 0.27 \\
\hline Verbal IQ & 14.2 & 13.1 & 0.88 & 0.69 \\
\hline Performance IQ & 12.4 & 16.6 & 1.84 & 0.03 \\
\hline \multicolumn{5}{|l|}{ CANTAB } \\
\hline MTS, total correct & 1.1 & 0.5 & 0.17 & $<0.001$ \\
\hline RTI & 1.1 & 0.5 & 0.18 & $<0.001$ \\
\hline \multicolumn{5}{|l|}{ 5-choice movement time } \\
\hline 5-choice reaction time & 1.1 & 0.4 & 0.11 & $<0.001$ \\
\hline RVPA & 1.1 & 0.4 & 0.12 & $<0.001$ \\
\hline \multicolumn{5}{|l|}{ SOC } \\
\hline Mean initial thinking time (5 moves) & 1.2 & 0.4 & 0.14 & $<0.001$ \\
\hline Mean subsequent thinking time (5 moves) & 1.0 & 1.0 & 0.97 & 0.94 \\
\hline Problems solved in minimum moves & 0.9 & 0.9 & 0.94 & 0.86 \\
\hline \multicolumn{5}{|l|}{ SWM } \\
\hline Between errors & 1.0 & 0.9 & 1.47 & 0.17 \\
\hline Strategy & 1.1 & 0.9 & 1.52 & 0.14 \\
\hline \multicolumn{5}{|l|}{ WCST } \\
\hline Perseverative errors & 1.1 & 0.5 & 0.20 & $<0.001$ \\
\hline Non-perseverative errors & 1.1 & 0.5 & 0.20 & $<0.001$ \\
\hline
\end{tabular}

Table 7 The relation between IQ and psychiatric diagnoses before and after adjustment for group status ${ }^{a}$

\begin{tabular}{|c|c|c|c|c|c|c|c|}
\hline \multirow[b]{2}{*}{ Psychiatric diagnosis } & \multirow[b]{2}{*}{$n$} & \multirow[b]{2}{*}{$\begin{array}{l}\text { Non-adjusted ORs } \\
(95 \% \mathrm{Cl})\end{array}$} & \multirow{2}{*}{$\begin{array}{l}\text { Children with } \\
\text { 22q11.2DS, stratified } \\
\text { ORs }(95 \% \text { Cl) }\end{array}$} & \multirow[b]{2}{*}{$\begin{array}{l}\text { Siblings, stratified } \\
\text { ORs }(95 \% \mathrm{Cl})\end{array}$} & \multirow[b]{2}{*}{$\begin{array}{l}\text { Adjusted ORs } \\
\text { (95\% Cl) }\end{array}$} & \multicolumn{2}{|c|}{ Homogeneity of ORs } \\
\hline & & & & & & $\chi^{2}$ & $P$ \\
\hline ADHD & 107 & $1.80(1.12-2.90)$ & $0.66(0.32-1.39)$ & $1.61(0.24-10.96)$ & $0.74(0.37-1.48)$ & 0.71 & 0.40 \\
\hline Any anxiety disorder & 108 & $1.90(1.08-3.35)$ & $1.40(0.62-3.15)$ & $0.47(0.07-3.23)$ & $1.19(0.56-2.51)$ & 1.04 & 0.31 \\
\hline ASD screening ${ }^{b}$ & 106 & $1.86(1.04-3.32)$ & $1.35(0.58-3.12)$ & $0.47(0.07-3.23)$ & $1.14(0.53-2.46)$ & 0.96 & 0.33 \\
\hline
\end{tabular}

\begin{tabular}{|c|c|c|c|}
\hline Psychiatric diagnosis & Indirect effect (mediated via IQ) $(95 \% \mathrm{Cl})$ & Direct effect $(95 \% \mathrm{Cl})$ & Total effect $(95 \% \mathrm{Cl})$ \\
\hline ADHD & $-0.10(-0.35$ to 0.13$)$ & 0.66 (0.31 to 0.93 ) & 0.56 (0.35 to 0.70$)$ \\
\hline Any anxiety disorder & $0.07(-0.17$ to 0.27$)$ & 0.38 (0.01 to 0.66 ) & 0.44 (0.20 to 0.63$)$ \\
\hline ASD screening $^{a}$ & $0.05(-0.25$ to 0.32$)$ & 0.39 (0.00 to 0.70$)$ & 0.44 (0.17 to 0.62$)$ \\
\hline
\end{tabular}

lack of evidence for a mediated effect of the deletion through cognition.

\section{Strengths and limitations}

This is one of the largest studies to date to include children with $22 \mathrm{q} 11.2 \mathrm{DS}$ with a relatively narrow age range as well as sibling controls, and one of the few studies to obtain detailed psychiatric as well as neurocognitive phenotyping. Moreover, this is the first study to use mediation analysis to further examine the relationships between IQ and psychopathology. Given that children with 22q11.2DS may have a risk of developing schizophrenia in adulthood in the order of $30 \%,{ }^{2,3}$ follow-up will allow us to identify risk and protective factors for schizophrenia and to study potential mechanisms and markers of high risk that are unconfounded by reverse causation and medication effects.

On the other hand, even though this study is one of the largest to date, type II errors cannot be excluded. Also, although the age range of the children is narrow in comparison to previous studies in children with 22q11.2DS, we cannot exclude the possibility that significant developmental changes during that period might have influenced our findings.

\section{Theoretical implications}

Our findings raise a number of important issues. First, it is not yet known how the deletion can manifest itself in such a clinically variable manner. Various factors could contribute to this 
variability, including the combined effect of reduced genetic dosage along with background genetic variation, in the $22 \mathrm{q} 11.2$ region, and elsewhere in the genome, as well as positional effects. ${ }^{33}$ For example, it has been hypothesised that dopamine dysregulation resulting from reduced gene dosage arising from the deletion could lead to brain disconnectivity and in turn to predisposition to the cognitive and psychiatric deficits for a subgroup of individuals with 22q11.2DS. ${ }^{34}$ Moreover, it is likely that environmental exposures also play a role.

These findings suggest that the 22q11.2 deletion has pleiotropic effects on IQ and psychopathology. ${ }^{9}$ In other words, the deletion has largely independent effects on IQ and risk of psychopathology. This implies that the associations seen more generally between impaired IQ and psychopathology might reflect the fact that cognitive impairments index genetic risk factors for psychiatric syndromes rather than that impaired cognition mediates the effects of risk. Indeed, there is accumulating evidence in support of this view from recent work on schizophrenia. ${ }^{35}$

\section{Clinical implications}

The results indicate that the prevalence of psychopathology in children with 22q11.2 DS is high, with over half meeting DSM-IV-TR criteria. The prevalent diagnoses, which are often comorbid in children with 22q11.2DS, include ADHD, anxiety disorders, ODD and high rates of autistic traits. The prevalence of intellectual disability is also high, with $31 \%$ of children with the deletion having mild intellectual disability. Moreover, children with 22q11.2DS seem to exhibit a generally impaired cognitive performance, which is more variable than in the non-deleted population (as indicated by larger standard deviations in the performance of children with $22 \mathrm{q} 11.2 \mathrm{DS}$ in relation to their siblings).

Furthermore, these findings underline the potential implications of childhood 22q11.2DS for parents and health services and indicate the importance of early detection and treatment. Our finding that psychopathology is not mediated by the children's intellectual impairment does not support the use of cognitive remediation for ameliorating the mechanisms underlying psychopathology in children with 22q11.2DS. However, our examination of general, rather than more specific cognitive functions, might have prevented the identification of relationships between specific cognitive deficits and psychiatric disorders.

These findings also provide no support for the suggestion that psychopathology in 22q11.2DS is a non-specific consequence of intellectual impairment reflecting mechanisms that differ from those operating in more typical cases. Rather, they are consistent with the conclusion that the mechanisms by which psychopathology is produced in 22q11.2DS might resemble those operating in more typical cases, and that 22q11.2DS might serve as a model in which mechanisms of childhood psychopathology might be studied.

In conclusion, we set out to determine the rate and nature of psychopathology and neurocognitive dysfunction, and the relationship between them, in children with 22q11.2DS. We found high rates of psychopathology and impairments across a wide range of neurocognitive functions compared with sibling controls. We found no evidence that the presence of psychopathology is correlated with neurocognitive impairment and it would appear that the former is not mediated by the latter. These findings have important clinical and theoretical implications. Further work is required to determine whether these findings can be extended to older age groups with 22q11.2DS, other CNVs and other high-risk samples.

\section{Funding}

This study was funded by the Baily Thomas Charitable Trust, the Waterloo Foundation and the Wellcome Trust.

\section{Acknowledgements}

We are extremely grateful to all the families that participated in this study as well as to our funders. We would also like to thank the following people for contributing to the study: Dr Jane Scourfield, Anna Pemberton, Aimee Davies, Elizabeth Mansell, Gregory Miller, Katherine Price, Jade Bowyer and Dr Davy Kavanagh.

Maria Niarchou, MSC, Stanley Zammit, MRCPsych, PhD, Institute of Psychological Medicine and Clinical Neurosciences, MRC Centre for Neuropsychiatric Genetics and Genomics, Cardiff University; Stephanie H. M. van Goozen, PhD, School of Psychology, Cardiff University; Anita Thapar, FRCPsych, PhD, Hayley M. Tierling $\mathrm{BSC}$, Michael J. Owen, FRCPsych, PhD, Marianne B. M. van den Bree, PhD, BSC, Michael J. Owen, FRCPsych, PhD, Marianne B. M. van den Bree, PhD,
Institute of Psychological Medicine and Clinical Neurosciences, MRC Centre for Neuropsychiatric Genetics and Genomics, Cardiff University, Cardiff, UK

Correspondence: Dr Marianne van den Bree, Institute of Psychological Medicine and Clinical Neurosciences, MRC Centre for Neuropsychiatric Genetics and Genomics, Cardiff University, Cathays, Maindy Road, Hadyn Ellis Building, Cardiff CF24 4HQ, UK. Email: vandenbreemb@cf.ac.uk

First received 14 May 2013, final revision 11 Aug 2013, accepted 15 Aug 2013

\section{References}

1 Bish JP, Chiodo R, Mattei V, Simon TJ. Domain specific attentional impairments in children with chromosome 22q11.2 deletion syndrome. Brain Cogn 2007; 64: 265-73.

2 Murphy KC, Jones LA, Owen MJ. High rates of schizophrenia in adults with velo-cardio-facial syndrome. Arch Gen Psychiatry 1999; 56: 940-5.

3 Bassett AS, Chow EWC, Husted J, Weksberg R, Caluseriu O, Webb GD, et al. Clinical features of 78 adults with $22 q 11$ deletion syndrome. Am J Med Genet A 2005; 138A: 307-13.

4 Green T, Gothelf D, Glaser B, Debbane M, Frisch A, Kotler M, et al. Psychiatric disorders and intellectual functioning throughout development in velocardiofacial (22q11.2 deletion) syndrome. J Am Acad Child Adolesc Psychiatry 2009; 48: 1060-8.

5 Shashi V, Veerapandiyan A, Schoch K, Kwapil T, Keshavan M, Ip E, et al. Social skills and associated psychopathology in children with chromosome 22q11.2 deletion syndrome: implications for interventions. J Intellect Disabil Res 2012; 56: 865-78.

6 Hooper SR, Curtiss K, Schoch K, Keshavan MS, Allen A, Shashi V. A longitudinal examination of the psychoeducational, neurocognitive and psychiatric functioning in children with 22q11.2 deletion syndrome. Res Dev Disabil 2013; 34: 1758-69.

7 Karayiorgou M, Simon TJ, Gogos JA. 22q11.2 microdeletions: linking DNA structural variation to brain dysfunction and schizophrenia. Nat Rev Neurosci 2010; 11: 402-16.

8 Walters JTR, Owen MJ. Endophenotypes in psychiatric genetics. Mol Psychiatry 2007; 12: 886-90.

9 O'Donovan MC, Kirov G, Owen MJ. Phenotypic variations on the theme of CNVs. Nat Genet 2008; 40: 1392-3.

10 Angold $A$, Prendergast $M$, Cox A, Harrington $R$, Simonoff $E$, Rutter $M$. The Child and Adolescent Psychiatric Assessment (CAPA). Psychol Med 1995; 25: 739-53.

11 American Psychiatric Association. Diagnostic and Statistical Manual of Mental Disorders (4th edn, text revision) (DSM-IV-TR). APA, 2000.

12 Rutter M, Bailey A, Lord C. Social Communication Questionnaire 2003. Western Psychological Services, 2003.

13 Wechsler D. Wechsler Abbreviated Scale of Intelligence (WASI) Manual. Psychological Corporation, 1999.

14 Heaton RK, Chelune GJ, Talley JL, Kay GG, Curtiss G. Wisconsin Card Sorting Test manual. Revised and Expanded. Psychological Assessment Resources, 1993.

15 Cohen J, Cohen P. Applied Multiple Regression/Correlation Analysis for the Behavioral Sciences. Erlbaum, 1983.

16 Kirkwood BR, Sterne AC. Essential Medical Statistics (2nd edn). Blackwell Science, 2003.

17 MacKinnon DP, Lockwood CM, Hoffman JM, West SG, Sheets V. A comparison of methods to test mediation and other intervening variable effects. Psychol Methods 2002; 7: 83-104. 
18 Royal College of Psychiatrists. Enabling People with Mild Intellectual Disabilities and Mental Health Problems to Access Healthcare Services (College Report CR175). Royal College of Psychiatrists, 2012.

19 Emerson E. Prevalence of psychiatric disorders in children and adolescents with and without intellectual disability. J Intellect Disabil Res 2003; 47: 51-8.

20 Antshel KM, Faraone SV, Fremont W, Monuteaux MC, Kates WR, Doyle A, et al. Comparing ADHD in velocardiofacial syndrome to idiopathic ADHD: a preliminary study. J Atten Disord 2007; 11: 64-73.

21 Jansen $P$, Duijff $S$, Beemer $F$, Vorstman J, Klaassen $P$, Morcus $M$, et al Behavioral problems in relation to intelligence in children with 22q11.2 deletion syndrome: a matched control study. Am J Med Genet A 2007; 143A: 574-80

22 Miller DT, Shen Y, Weiss LA, Korn J, Anselm I, Bridgemohan C, et al. Microdeletion/duplication at 15q13.2q13.3 among individuals with features of autism and other neuropsychiatric disorders. J Med Genet 2009; 46: 242-8.

23 Tranfaglia MR. The psychiatric presentation of fragile $x$ : evolution of the diagnosis and treatment of the psychiatric comorbidities of fragile $X$ syndrome. Dev Neurosci 2011; 33: 337-48.

24 Langley K, Martin J, Agha SS, Davies C, Stergiakouli E, Holmans P, et al. Clinical and cognitive characteristics of children with attention-deficit hyperactivity disorder, with and without copy number variants. Br J Psychiatry 2011; 199: 398-403.

25 Rommelse NN, Altink ME, Oosterlaan J, Buschgens CJ, Buitelaar J, Sergeant JA. Support for an independent familial segregation of executive and intelligence endophenotypes in ADHD families. Psychol Med 2008; 38: 1595-606.

26 Fry AF, Hale S. Processing speed, working memory, and fluid intelligence: evidence for a developmental cascade. Psychol Sci 1996; 7: 237-41.

27 Niarchou M, Zammit S, Walters J, Lewis G, Owen MJ, van den Bree MBM. Defective processing speed and nonclinical psychotic experiences in children: Iongitudinal analyses in a large birth cohort. Am J Psychiatry 2013; 170: $550-7$.

28 Rockers K, Ousley O, Sutton T, Schoenberg E, Coleman K, Walker E, et al Performance on the Modified Card Sorting Test and its relation to psychopathology in adolescents and young adults with 22q11.2 deletion syndrome. J Intellect Disabil Res 2009; 53: 665-76.

29 Lewandowski KE, Shashi V, Berry PM, Kwapil TR. Schizophrenic-like neurocognitive deficits in children and adolescents with 22q11 deletion syndrome. Am J Med Genet B Neuropsychiatr Genet 2007; 144: 27-36.

30 De Smedt B, Devriendt K, Fryns JP, Vogels A, Gewillig M, Swillen A. Intellectual abilities in a large sample of children with Velo-Cardio-Facial Syndrome: an update. J Intellect Disabil Res 2007; 51: 666-70.

31 Moss EM, Batshaw ML, Solot CB, Gerdes M, McDonald-McGinn DM, Driscoll DA, et al. Psychoeducational profile of the 22q11.2 microdeletion: a complex pattern. J Pediatr 1999; 134: 193-8.

32 Antshel KM, Fremont W, Roizen NJ, Shprintzen R, Higgins AM, Dhamoon A et al. ADHD, major depressive disorder, and simple phobias are prevalent psychiatric conditions in youth with velocardiofacial syndrome. J Am Acad Child Adolesc Psychiatry 2006; 45: 596-603.

33 Williams NM. Molecular mechanisms in 22q11 deletion syndrome. Schizophr Bull 2011; 37: 882-9.

34 Karayiorgou M, Simon TJ, Gogos JA. 22q11.2 microdeletions: linking DNA structural variation to brain dysfunction and schizophrenia. Nat Rev Neurosci 2010; 11: 402-16.

35 Fowler T, Zammit S, Owen MJ, Rasmussen F. A population-based study of shared genetic variation between premorbid IQ and psychosis among male twin pairs and sibling pairs from Sweden. Arch Gen Psychiatry 2012; 69: 460-6. 